\title{
BEYOND TCP/IP: A CONTEXT-AWARE ARCHITECTURE
}

\author{
Guy Pujolle ${ }^{1}$, Hakima Chaouchi ${ }^{1}$, and Dominique Gaiit ${ }^{2}$ \\ ${ }^{\prime}$ LIP6-University of Paris 6, 8 rue du Capitaine Scott, 75015 Paris, France; ${ }^{2}$ ISTIT - M2S \\ team-UTT, 12 rue Marie Curie - 10000 Troyes, France
}

\begin{abstract}
To configure current networks a large number of parameters have to be taken into account. Indeed, we can optimize a network in different ways: Optimize battery capacity, optimize reliability of the network, optimize QoS, optimize the security, optimize the mobility management, and so on. In this paper we begin by proving that the TCP/IP architecture is not the best protocol in a specific wireless experiment: the energy consumption on a Wi-Fi network. Then, we propose a new architecture, Goal-Based Networking (GBN) architecture, using adaptable protocols named STP/SP protocols (Smart Transport Proto$\mathrm{col} /$ Smart Protocol) able to optimize locally the communications through the networks. Finally we discuss the pros and cons of this new architecture.
\end{abstract}

Key words: TCP/IP, energy consumption, wireless networks, protocol, goal-based management.

\section{INTRODUCTION}

Wireless communications are more and more involved in different applications, and are already part of our daily lives. These new wireless technologies are growing faster than the networking technologies. In fact, the basic networking technology is TCP/IP communication model. This model has been designed in wired networks. Wireless networks requirements and applications were not considered during the design of this model. TCP/IP model is facing a limitation in wireless and mobile environment ${ }^{1-3}$. This limitation could become important in future wireless environments ${ }^{4 \cdot 6}$. In this paper, we illustrate via Wi-Fi network measurements on energy consumption the non efficiency of TCP/IP. This proves the need for a new architecture that includes a smart mechanism able to decide which communication proto- 
col is suitable for a certain situation (Goal) in the network, and also adapt the parameters of the selected protocols to better react to the present and future state of the network.

The rest of the paper is organized as follows. First we illustrate via a WiFi measurement the problems of the TCP/IP stack. Then, we introduce the new STP/SP model, followed by the description of the smart architecture (Goal Based Networking architecture) to support the deployment of the new STP/SP model. Finally, we present an analysis of this architecture and we conclude this work.

\section{TCP/IP LIMITATION IN WI-FI NETWORKS}

We are looking in this section at a Wi-Fi network and we are interested in measuring energy consumption in different experiences. We measured a file transmission on a Wi-Fi network. The transmission was at a $100 \mathrm{~mW}$ level and the receiver was at 2 meters in a direct view so that the quality of the signal is sufficient to avoid retransmission at the MAC layer. We transmit a $100 \mathrm{Mbytes}$ file fragmented into 100 bytes TCP/IP packets. To send one useful bit of the payload on a Wi-Fi wireless network of a TCP/IP packet, we got the energy of approximately $700 \mathrm{~nJ}$.

As a cycle of the processor asks for approximately $0,07 \mathrm{~nJ}$, the transmission for one bit of the payload is approximately equal to 10000 cycles of the processor.

When measuring the consumption of just one bit out of the 100 Mbytes, we obtained $70 \mathrm{~nJ}$ for the transmission of one bit. Therefore, we can deduce that the TCP/IP environment is asking on the average 10 times more energy to transmit one useful bit than for the transmission of one bit. It has to be noted that the transmission of small IP packets asks for a large number of small signalling packets. This explains in part the high energy consumption. Another part of the energy consumption comes from the number of overhead bits produced by the TCP/IP architecture.

Several other experiments on sensor networks lead to the same outcomes.

Two significant conclusions can be provided from these measurements:

- The TCP/IP protocol over Wi-Fi and more generally over wireless systems is very energy consuming when the segmentation provides small packets.

- As it is necessary to send ten bits for one efficient bit, the question is: is it possible to find another protocol able to improve the energy consumption? 
It turns out that TCP/IP is not an efficient protocol for wireless networks as soon as small packets have to be sent. This is almost expected since TCP/IP was designed to cope with wired networks.

On this example, we have shown that the TCP/IP is not very efficient for wireless networks. We can find other examples where the TCP/IP architecture is not optimal at all on QoS, reliability or security issues. The sequel of this paper is a proposal for a new architecture able to optimize not only the energy but also different performance parameters.

\section{A NEW SMART ARCHITECTURE STP/SP}

TCP/IP architecture was created for the interconnection of networks running with different architectures. Then, the TCP/IP architecture was chosen as the unique architecture for all communications. The advantage is clearly to permit a universal interconnection scheme of any kind of machines. However, TCP/IP is only a tradeoff and we wonder if specific architectures but, may be, IP compatible could not be a better solution to optimize the communications. The idea is to propose a Smart Protocol (SP) that can adapt to the environment, for optimizing battery or optimizing reliability or optimizing QoS or any other interesting functionality. The design of a Smart Protocol at the network layer that is aware of the upper and the lower layers and adapts their communication to a set of parameters is obviously the ultimate communication architecture that can support current and emerging wireless networks. This new context-aware architecture that we named STP/SP Smart Transport Protocol/Smart Protocol could be compatible with IP.

Indeed, the SP protocol is a set of protocols SP1, SP2, ....SPn that could be either derived from the IP protocol or could be adapted to specific environments. In the same way the STP protocol is a set of protocol that could be derived from the TCP protocol or from independent protocols. In this paper, we are interested in the compatibility of STP/SP with the TCP/IP architecture. Indeed, the TCP/IP functionalities are rich enough to cope with the different situations.

All the different architectures are easily interconnected through a classical TCP/IP protocol. For instance, a sensor network will deploy its STP/SP protocol stack that support the requirements of the application set up over the sensor network. This sensor network will be interconnected through a classical TCP/IP gateway to another network that deploys another STP/SP protocol stack which supports the requirements of this other network. This might sound as going back to the period where the networks deploy their proprietary protocols. Then, IP was designed to interconnect these networks. Next IP was generalized and today reached the point where this protocol 
cannot cope with all types of environment such as wireless environments. The difference between the STP/SP approach and the former proprietary solutions is that STP/SP will basically use the TCP/IP concepts and functionalities, but in a smart way. In fact, rather than deploying TCP/IP in the same way in any environment without being aware of the requirements of this environment, STP/SP will offer a smart TCP/IP like environment. This will keep the simplicity and efficiency of TCP/IP, but will add a smart process that is totally absent in TCP/IP. This smart process will be deployed using a new architecture in the network guided by a set of objectives named Goals.

We describe this global architecture in Figure 1. The objective of this architecture is to implement the smart process of selecting the sub-protocol of the STP/SP protocol that fulfils the requirements of the concerned network. This is a goal-based networking architecture and the control is a goal-based control.

\section{A GOAL-BASED NETWORKING ARCHITECTURE}

The goal-based architecture is composed of mainly two mechanisms: The smart mechanism to select the STP/SP protocol and its parameters, and the enforcement mechanism to enforce the decisions of the smart mechanism. We propose to use agent-based mechanism to implement the smart mechanism, and to use some concepts of the policy based networking ${ }^{7}$ such as the enforcement procedures to implement the enforcement mechanism.

An agent-based platform permits a meta-control structure such as the platform described in $^{8}$. Assuming that for each network node we associate one or several agents, the network can be seen as a multi-agent system in which the main goal is to decide about the control to use for optimizing a given functionality described in the goal distributed by the meta-agent.

Intelligent agents are able to acquire and to process information about situations that are "not here and not now", i.e., spatially and temporally remote. By doing so, an agent may have a chance to avoid future problems or at least to reduce the effects. These capabilities allow agents to adapt their behavior according to the traffic flows going through the node.

Finally, interruptible agents may be used. They are able to act intelligently and to respond in real time. This capability allows implementing complex mechanisms able to take care of real time decisions.

It is important to note that other works has proposed a decision mechanism in the network to enforce decision or policies in the network. This typical architecture named Policy-based Networking (PBN) enforces high level decisions without unfortunately considering the problem optimization of pa- 
rameters related to lower levels of the network. It's only a top down approach. In our proposed architecture, we intend to use the enforcement procedure of policy-based networking architecture that is an interesting concept for automating the enforcement of the smart mechanism decisions. The Goal-based architecture considers the optimizing problem related to the higher but also the lower layers of the network, and enforces the most suitable STP/SP protocols and parameters for the given network and application.

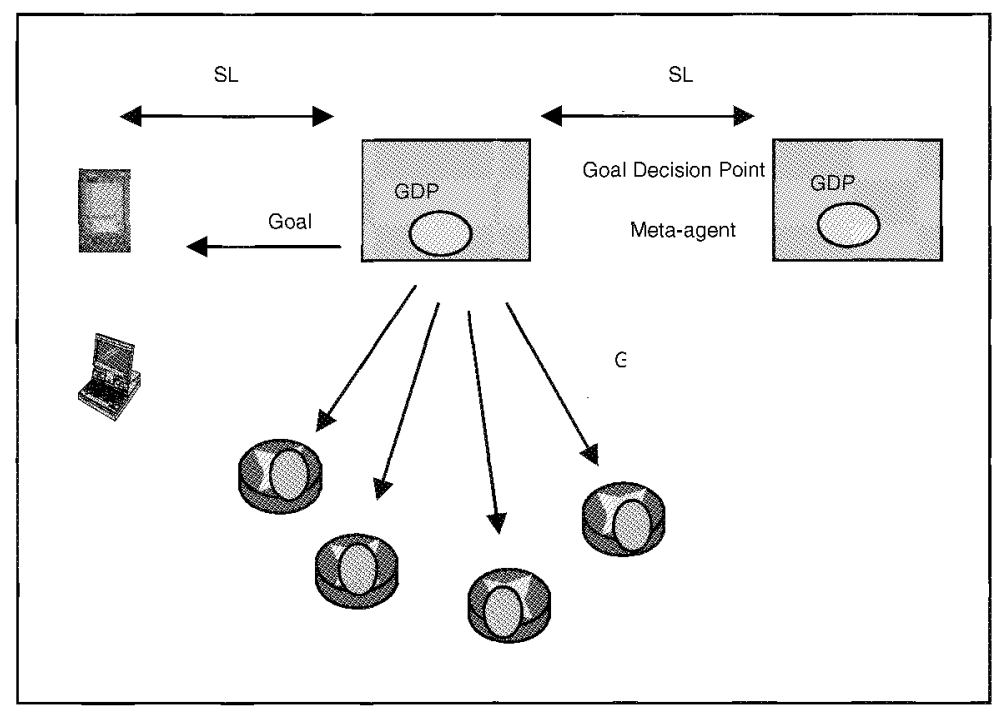

Figure 1. The global architecture.

\section{THE PROPOSED GOAL-BASED ARCHITECTURE}

Figure 1 depicts the global Goal-based architecture (GBN) and Figure 2 depicts the GBN and STP/SP reference model.

First, users can enter their SLA through a Web service scheme for example. The manager of the network can also enter the network configurations corresponding to the goals of the network. A meta-agent in the smart Layer is able to decide about the global goal of the network. This meta-agent is supported by any kind of centralized servers if any. As soon as defined, the goal is distributed to the different network nodes or Goal Enforcement Point (GEP). Knowing the goal, the different nodes have to apply policies and then configuration to reach the goal. 
The agents in the GEP are forming a multi-agent system. The problem is to group these agents for providing a global task. The Distributed Artificial Intelligence (DAI) systems may be seen as such a grouping.

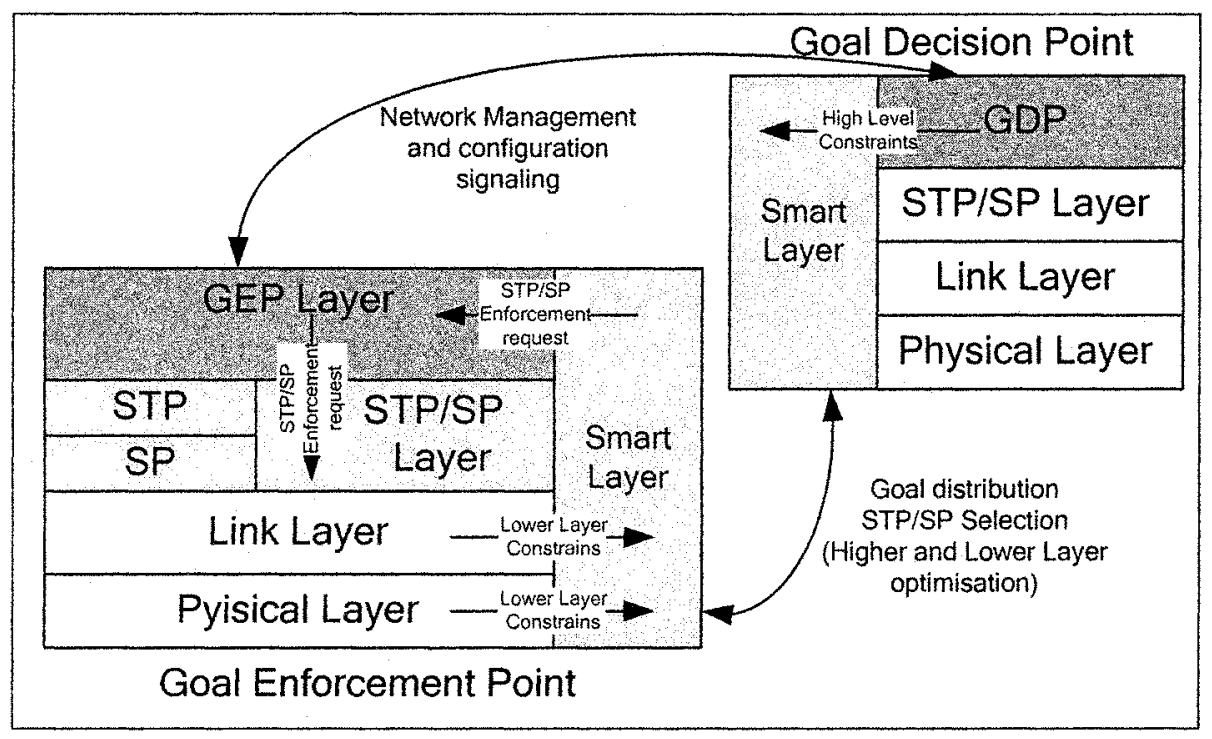

Figure 2. GBN and STP/SP reference Model

The Smart Layer is in charge of collecting the different constraints from the lower layers but also from the higher layer (business level policies), then specify and update the goal of the network which is about what to optimise in the network and what to be offered by the network. Note that the classical approaches consider only, what to be offered by the network. After specifying the network goal, the smart layer selects the STP/SP protocols and parameters that will optimize the specified goal. The smart layer will keep updating the goal of the network based on the current state of the network or on a new policies introduced by the Goal Decision Point.

The smart Layer can be implemented by an agent-based architecture. It is very suitable to provide the specification of the network goal and also the selection of the suitable STP/SP protocols.

The choice of the protocol can be seen at two levels: the local and the global level. One agent in each node (Smart Layer) may be defined for deciding the local protocol in cooperation with the other agent of the multiagent system. Each agent has to perform a specific procedure, which is triggered according to the state of the node, to the QoS required, and to any other reason. This constitutes a local level for the decision. Moreover, agents 
can periodically interact to exchange their knowledge and ask to other agents if they need information they do not have. This constitutes the global level.

The smart layer interacts with the Goal Enforcement point (GEP) in order to enforce the STP/SP selected protocol that realizes the global goal. This implies also the definition of the algorithms to manage the CPU, the sensor, the radio or any parameter of the traffic conditioner.

Indeed, the traffic conditioner is replaced by an extended traffic conditioner (XTC) where different algorithms can be supported. The GEP is in charge to decide the value of the parameters and to decide about the protocol to be used. Within the entities that can be configured, classical droppers, meters, schedulers, markers, etc. may be found but also resource of the battery, availability, security parameters, radio parameters, etc. This XTC is shown in Figure 3.

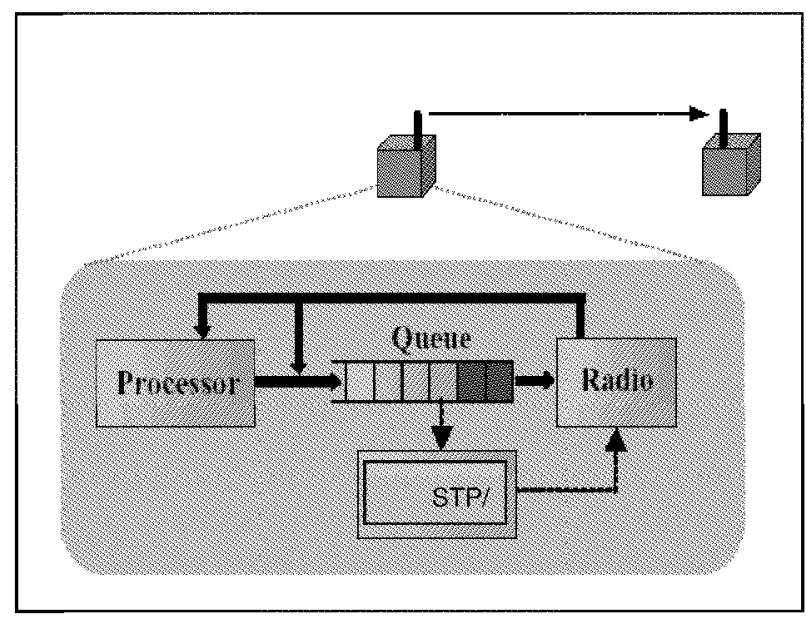

Figure 3. The Extended Traffic Conditioner XTC

\section{GENERALISED GOAL-BASED ARCHITECTURE}

We described in section 3, the global architecture. The agents managing the Goal Enforcement Point have to be installed in the nodes of the networks. However, an important part of the equipment is the end terminal. This terminal could be either powered or not. So, the routers cannot decide about the best protocol to use without knowing some characteristics of the terminal. If, for example, the terminal is a wireless terminal with low-level batteries, the goal of the network could be to take care of the consumption of the batteries and to decide to optimize the lifetime of the system. So it is 
necessary to implement a Goal Enforcement Point (GEP) in the terminal equipment. This architecture is presented in Figure 4.

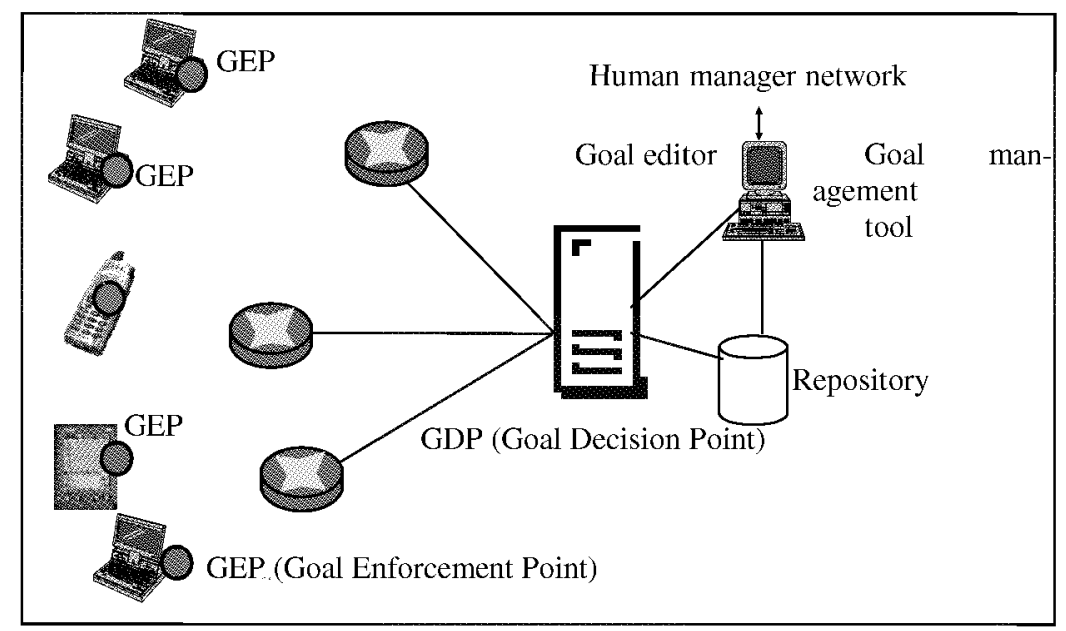

Figure 4. The Goal Based Networking Architecture

The GEP supports an agent that is part of the global multi-agent system. The agent receives from the Goal Decision Point the goal to apply. The goal received by the GEP has to be implemented through the agent. So, the agent using the relationship with the other agents of the multi-agent system has to choose the algorithms to use and particularly what STP/IP protocol is appropriate to optimize the goal. For example, if the terminal is a small portable or a sensor, the optimization can concern the energy. The agents of the multiagent system are communicating through small messages to be coordinated. The agent is also deciding about the values of the different parameters of the protocol. For security reasons the agent is stocked in a smartcard connected to the terminal as an USB key for example. The user cannot modify the agent and the goal due to the restricted access to the card. The smartcard is also a way to authenticate the user and to apply a goal that could be dependent of the profile of the user memorized in the smartcard. The architecture with the smartcard is shown in Figure 5.

Two kinds of agents are defined in our architecture:

- The meta-agent which monitors the other agents that sends goal and receives alarms;

- Intelligent cooperative agents deciding about protocols to be used and monitoring local parameters of the extended traffic controller.

We can distinguish two levels of decision within a network node. These two levels are the following: 
- A control mechanism at the lower level. This level is composed of the different node control mechanisms currently activated. Each control mechanism has its own parameters, conditions and actions, which can be monitored and manipulated by the entity lying at the higher level, the meta-agent. The functioning of a control mechanism is limited to the execution of the loop (goal and condition $\rightarrow$ actions). This is realized by a reactive agent. There is a causal link between the different stimuli (events) that a control mechanism recognizes and the actions it executes.

- The meta-agent chooses the goals to undertake by consulting the current system state (nodes state, requested SLA, goal of the company) and goals memorized in a goal repository. The meta-agent activates goals.

The nodes, thanks to the two control levels, respond to internal events (loss percentage for a class of traffic, load percentage of a queue, etc.) and external ones (a message sent by a neighbor node, reception of a new goal, etc.).

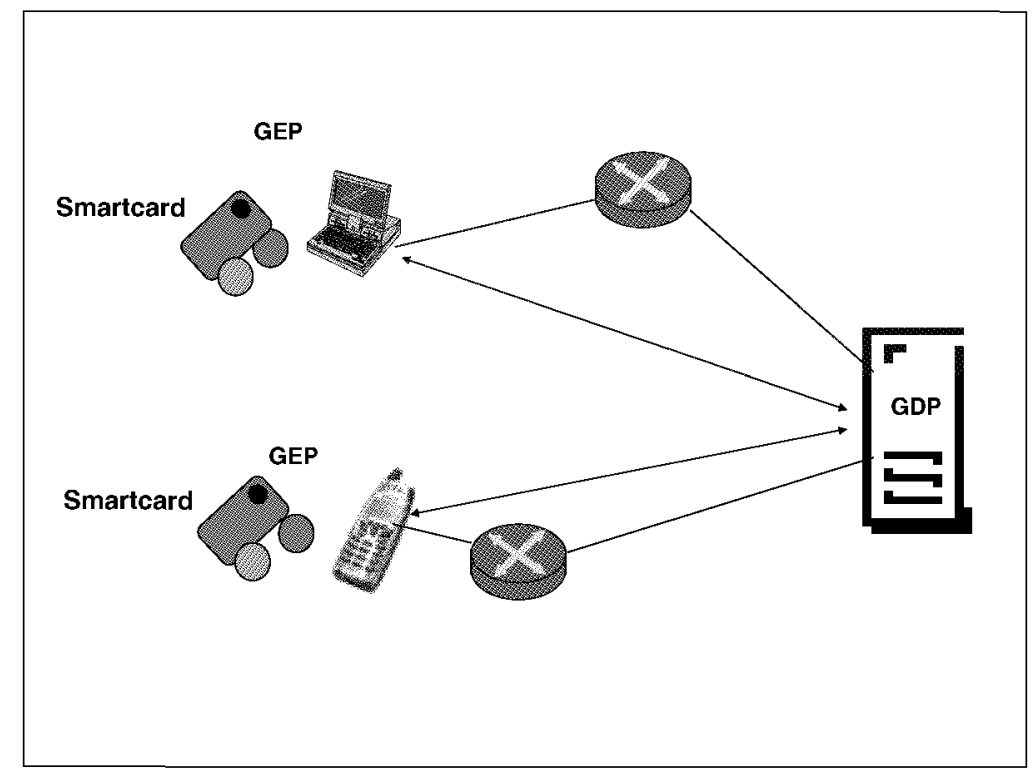

Figure 5. The architecture with the smartcard

\section{CONCLUSIONS}

This paper introduced a new communication architecture to better support wireless networks and new applications. STP/SP (Smart Transport Protocol/Smart Protocol) is a smart communication model that will use different 
transport and network protocols for each wireless environment. These protocols consider not only the policies provided by the business plan but also the constraints of the lower layers of the network. A smart architecture is proposed to provide the selection mechanism of the corresponding protocols and parameters. This smart architecture interacts with and enforcement architecture in order to configure the network with the selected protocols and parameters. A first analysis of our architecture, shows that smart selection of the communication protocols to use for a certain network environment and application bring better results than the classical TCP/IP architecture. Further research intend to focus on different wireless networks environments and design the STP/SP corresponding architecture that optimizes all the lower and higher layers parameters. The first considered environment is sensor networks.

\section{REFERENCES}

1. A. Boulis, P. Lettieri, Mani B. Srivastava, Active Base Stations and Nodes for Wireless Networks, Wireless Networks 9(1), 37-49 (2003).

2. C. Schurgers, V. Tsiatsis, S. Ganeriwal, M. B. Srivastava, Optimizing Sensor Networks in the Energy-Latency-Density Design Space, IEEE Transactions on Mobile Computing 1(1), 70-80 (2002).

3. P. Lettieri, C. Schurgers, Mani B. Srivastava, Adaptive link layer strategies for energy efficient wireless networking, Wireless Networks 5(5), 339-355 (1999).

4. C. E. Jones, K. M. Sivalingam, P. Agrawal, and J. C. Chen, A survey of energy efficient network protocols for wireless networks, Wireless Networks, 7(4), 343-358 (2001).

5. M. Stemm and R. H. Katz, Measuring and reducing energy consumption of network interfaces in hand-held devices, IEICE Transactions on Communications, E80-B(8), 1125 1131 (1997).

6. C. E. Jones, K. M. Sivalingam, P. Agrawal, and J. C. Chen, A survey of energy efficient network protocols for wireless networks, Wireless Networks, 7(4), pp. 343-358, July 2001.

7. D. C.Verma, Simplifying Network administration using policy-based management, IEEE Network 16(2), (2002).

8. D. Gaïti, and G. Pujolle, Performance management issues in ATM networks: traffic and congestion control, IEEE/ACM Transactions on Networking, 4(2), (1996). 